\title{
BMJ Open WeChat-based health education to improve health knowledge in three major infectious diseases among residents: a multicentre case- controlled protocol
}

\author{
Yan Qiu (D) , ${ }^{1}$ Hongli Qin, ${ }^{1}$ Meike Ying, ${ }^{1}$ Kaijin Xu, ${ }^{2}$ Jingjing Ren ${ }^{1}$
}

To cite: Qiu Y, Qin $\mathrm{H}$, Ying $\mathrm{M}$, et al. WeChat-based health education to improve health knowledge in three major infectious diseases among residents: a multicentre casecontrolled protocol. BMJ Open 2020;10:e037046. doi:10.1136/ bmjopen-2020-037046

- Prepublication history for this paper is available online. To view these files, please visit the journal online (http://dx.doi org/10.1136/bmjopen-2020037046).

Received 16 January 2020 Revised 22 September 2020 Accepted 03 October 2020

Check for updates

(c) Author(s) (or their employer(s)) 2020. Re-use permitted under CC BY-NC. No commercial re-use. See rights and permissions. Published by BMJ.

${ }^{1}$ General Practice Department, The First Affiliated Hospital, School of Medicine, Zhejiang University, Hangzhou, China ${ }^{2}$ State Key Laboratory for Diagnosis and Treatment of Infectious Diseases, National Clinical Research Center for Infectious Diseases, Collaborative Innovation Center for Diagnosis and Treatment of Infectious Diseases, The First Affiliated Hospital, School of Medicine, Zhejiang University, Hangzhou, China

Correspondence to

Dr Jingjing Ren;

3204092@zju.edu.cn

\section{ABSTRACT}

Introduction Health literacy $(\mathrm{HL})$ in infectious diseases is inadequate in China. Since the first nationwide survey of $\mathrm{HL}$ conducted in China, great efforts have been made. However, the rate of $\mathrm{HL}$ in infectious diseases was $16.06 \%$ in 2017. In contrast, with an $\mathrm{HL}$ rate of $15.85 \%$ in 2008 , no significant effect was observed over 10 years. With an increasing number of internet users, we aim to assess the effects of WeChat-based health education for the promotion of partial HL-health knowledge in infectious diseases.

Methods and analysis A total of 2160 residents aged 15-69 years old will be enrolled in this study. The primary outcome measures will be the rate of health knowledge in infectious disease. The follow-up period is 3 years.

Ethics and dissemination The study protocol was approved by the Research Ethics Committee of the First Affiliated Hospital, College of Medicine, Zhejiang University. The findings of this study will be submitted to a peerreviewed journal.

\section{INTRODUCTION}

According to $\mathrm{WHO}$, viral hepatitis causes 1.4 million deaths annually, being the secondleading killer infectious disease after tuberculosis, and nine times more people are infected with hepatitis than with HIV. $^{1}$ Viral hepatitis B, tuberculosis and HIV/AIDS are three common infectious diseases in China. ${ }^{2}$ In 2008, national health literacy (HL) investigations were first conducted, and the rate of HL was $15.85 \%$ for infectious diseases in China. $^{3}$ Although great efforts had been made, the rate of HL was $16.06 \%$ in 2017 , reflecting a slight increase over ten years. ${ }^{4}$ In the era of the internet, an increasing number of residents are increasingly inclined to use social media to seek online health information. China has witnessed a dramatic increase in internet users who have ever used the internet in the last year. For instance, the percentage of internet users increased from
Strengths and limitations of this study

- This study is a multicentre case-controlled study to assess the effect of WeChat-based health education to improve health knowledge in three infectious diseases in China.

- A previous study has identified the positive effect of WeChat-based health education. As WeChat is the most popular app used in China, an individual may have a greater chance of being involved in WeChatbased health education.

- Health knowledge is reported by residents themselves, which could lead to bias due to overestimation or underestimation.

$45.8 \%$ of the general population in 2013 to $55.8 \%$ in $2017 . .^{56}$

In China, WeChat is the most popular mobile app that provides social media services, similar to Facebook in Western countries. Previous studies have determined the positive effect of WeChat-based health education. ${ }^{78}$ In our previous study, nearly half $(40.6 \%)$ of internet users reported having sought health information via the internet. ${ }^{9}$ The proportion of internet users was higher than $32.9 \%$ in Vietnam; these individuals used the internet to obtain health information. ${ }^{10}$ Hence, we aim to assess the effect of WeChat-based health education in infectious diseases among residents.

\section{Study objectives}

We aim to assess the effects of WeChatbased health education for the promotion of health knowledge in infectious diseases. We hypothesise that WeChat-based health education could improve health knowledge in infectious diseases among residents in China. 
Table 1 Distribution of sex and age in this study

720 participants in each group

\begin{tabular}{lccc}
\cline { 2 - 4 } Age (years) & Male & Female & Total \\
\hline 15 & 31 & 26 & 57 \\
20 & 39 & 35 & 74 \\
25 & 51 & 49 & 99 \\
30 & 41 & 41 & 82 \\
\hline 35 & 38 & 37 & 75 \\
40 & 45 & 43 & 88 \\
45 & 50 & 48 & 98 \\
50 & 46 & 45 & 91 \\
55 & 28 & 27 & 56 \\
\hline Total & 369 & 351 & 720 \\
\hline
\end{tabular}

The total numbers of adults in the 25 years and 55 years categories are rounded.

\section{METHODS AND ANALYSIS \\ Design}

This study is designed as a prospective case-control study, with an allocation of 1:1.

\section{Settings and participants}

The study will be conducted in six districts in Zhejiang Province, China, from 1 January 2018 to 31 December 2020. The six districts are selected according to economic levels. Higher economic-level areas are Tongxiang and Dinghai, intermediate economic-level areas are Putuo and Putuoshan in Zhoushan, and lower economic-level areas are Nanxun and Tonglu. We aim to recruit adult residents in these six settings using probability proportional to size sampling. In addition, the distribution in sex and age of participants is shown in table 1 according to the National Bureau of Statistics 2017. ${ }^{11}$

\section{Eligibility criteria}

Residents have to fulfil all of the following criteria to be eligible for the study: age between 15 and 69 years; sign informed consent. The exclusion criteria are as follows: children and adolescents younger than 15 years; elderly adults older than 60 years; the presence of psychiatric diseases; and unwillingness to participate in the study.

\section{Interventions}

The intervention in the study is WeChat-based health education. Figure 1 shows an example, and table 2 is the translated version. There are two intervention groups. Group I will be given WeChat-based health education. Group II will be given traditional plus WeChat-based health education. The control group is only given traditional health education, such as clinic education and pamphlet education. A total of 2160 participants are randomly divided into groups I and II and the control group in six districts.

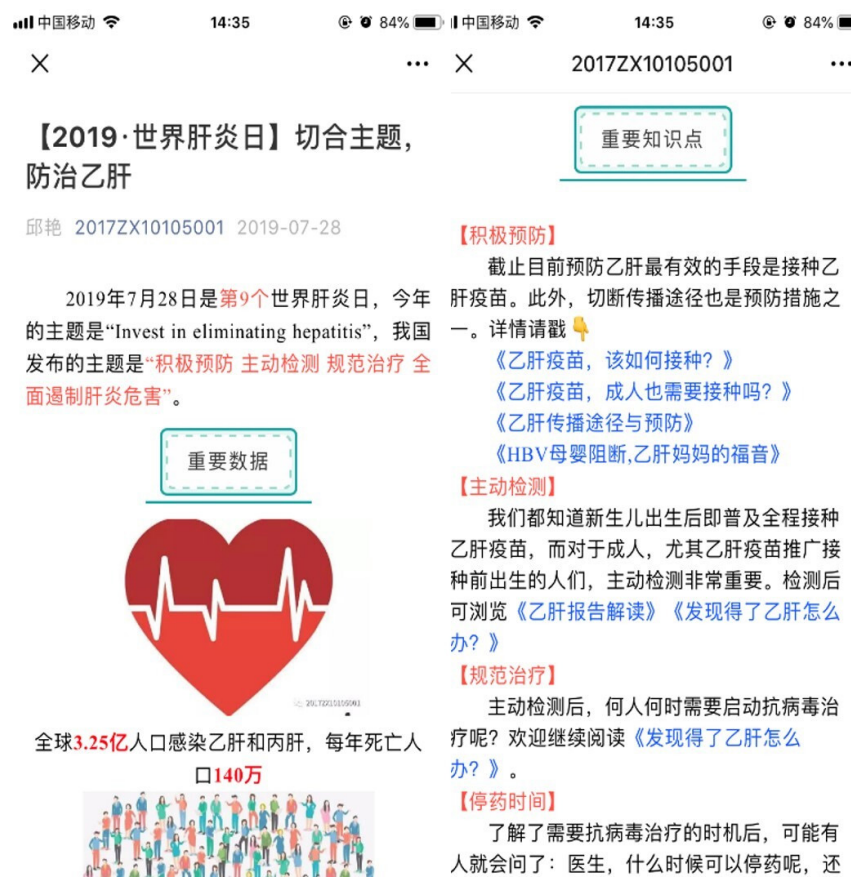

Figure 1 Partial screening of the hepatitis B sheet published on the WeChat platform (official number for health education: 2017ZX10105001).

\section{Study procedure}

Oral and written information regarding the study will be first delivered to the participants and signed by them. Participants in groups I and II will receive WeChat-based health education once or twice monthly. The theme is around hepatitis B, tuberculosis and HIV/AIDs. The effect will be assessed through a self-designed questionnaire in our previous study (table 3 ). To ensure quality, the questionnaire must be verified by the quality controller on the same day.

\section{Follow-up}

All the participants will report baseline rate of health knowledge in infectious disease and those after a 3-year intervention.

\section{Outcome measures}

The primary outcome is the rate of health knowledge in infectious disease in residents.

\section{Sample size}

The HL level of infectious disease was $17.12 \%$ in 2013 in China. To detect a $15 \%$ difference between groups while maintaining a power of $80 \%$ and a significance level of $5 \%$ and allowing for $10 \%$ of the residents will be lost to follow-up, a total of 2160 participants will be needed.

\section{Data collection and statistical analysis}

All the participants will be assigned a study number. After the collection of the completed paper questionnaire, data will be double entered into an Epidata V.3.1 software database by two staff members independently. Then, we will check the accuracy, consistency and logicality of the data. 
Table 2 Translation of figure 1

\section{(2019 World Hepatitis Day) The theme to prevent hepatitis B}

28 July 2019 , is the ninth

World Hepatitis day.

The theme is 'Invest in eliminating hepatitis'. In China the theme is 'Active Prevention, Active Testing, Standardised Treatment and Comprehensive Control of Hepatitis Harm'.

\section{(Important Data)}

Viral hepatitis $B$ and $C$ affect 325 million people worldwide causing 1.4 million deaths a year.

\section{0 people became} newly infected in 2017.

Over $\mathbf{8 0} \%$ of people living with hepatitis lack prevention, testing and treatment services.

\section{(Key points)}

\section{(Active Prevention)}

So far, the most effective way to prevent hepatitis $B$ is to be inoculated with the hepatitis $B$ vaccine. In addition, cutting off the route of transmission is one of the preventive measures. Please click the following:

'How to get the hepatitis $B$ vaccine?'

'Do adults also need to get the hepatitis B vaccine?'

'Transmission route and prevention of hepatitis B' 'Block the mother-to-child transmission route of HBV, the gospel for mothers with hepatitis B'

$\mathrm{HBV}$, hepatitis B virus.

The SPSS software V.22.0 is used for data processing and analysis. Descriptive statistics will be used to summarise baseline characteristics. Student's t-test will be used to compare the mean values of continuous variables approximating a normal distribution. For non-normally distributed variables, the Mann-Whitney $U$ test will be used. The $\chi^{2}$ test or Fisher's exact test will be used, as appropriate, to compare percentages. The difference between study groups will be considered significant when the $P$ value is below 0.05 . All statistical hypothesis tests will be two tailed.

\section{Harms}

As the study does not involve blood collection or drug treatment, there are no harms in this study.
Table 3 Self-designed questionnaire

\section{Items Contents}

Knowledge

Do you know that hepatitis B virus (HBV) is contagious?

How does HBV spread?

What is the most effective method to prevent the spread of HBV?

Should newborns be vaccinated against HBV within 24 hours after birth?

Do you know that the disease progression of the majority of hepatitis B patients can be controlled after antiviral treatment?

Could you recognise a person infected with HIV by appearance?

How does HIV spread?

Which measures can reduce the spread of AIDS?

How does tuberculosis spread?

What symptoms should indicate the presence of tuberculosis?

What are the measures to prevent the spread of tuberculosis?

\section{Attitude and practice}

Would you like your family members to receive the hepatitis $B$ vaccine?

Have you received the hepatitis $B$ vaccine?

Do you have a habit of sharing toothbrushes, toothpicks or razors with others?

Do you use condoms during sex? (if no history of sex, no answer required)

Will you cover your face when you cough or sneeze?

Do you often open windows for ventilation at home?

Skills

Can you quickly obtain knowledge about hepatitis B, HIV/ AIDS and tuberculosis through the internet or newspapers?

Can you understand drug instructions?

Can you understand health education materials about hepatitis B, AIDS or tuberculosis, such as pictorials and brochures?

\section{Auditing}

The Ethics Committee does not require auditing for this study.

\section{Ethics and dissemination}

All the participants have to sign written information regarding informed consent. The full protocol will be freely available due to open access publication. The findings of this study will be submitted to a peer-reviewed journal. The Strengthening the Reporting of Observational Studies in Epidemiology statement will be followed for this case-controlled study.

Acknowledgements The authors would like to thank AJE for helping to revise the language. In addition, we appreciate the support of the staff from districts of Nanxun, Tonglu, Tongxiang, Dinghai, Putuo and Putuoshan in Zhejiang Province.

Contributors $Y Q$ conceptualised the study. $Y Q$ developed the first draft of the manuscript. YQ, HQ, MY, KX and JR contributed to the development of the study 
protocol and approved the final draft of the manuscript. $Y Q$ and JR are the guarantor.

Funding This study is funded by the National Scientific and Technological Major Project of China (2017ZX10105001).

Competing interests None declared.

Participant and public involvement No participants or the public will be involved in the design, or conduct, or reporting, or dissemination plans of this research.

Patient consent for publication Not required.

Ethics approval The Research Ethics Committee of the First Affiliated Hospital,College of Medicine, Zhejiang University (No: 2017-729).

Provenance and peer review Not commissioned; externally peer reviewed.

Open access This is an open access article distributed in accordance with the Creative Commons Attribution Non Commercial (CC BY-NC 4.0) license, which permits others to distribute, remix, adapt, build upon this work non-commercially, and license their derivative works on different terms, provided the original work is properly cited, appropriate credit is given, any changes made indicated, and the use is non-commercial. See: http://creativecommons.org/licenses/by-nc/4.0/.

ORCID iD

Yan Qiu http://orcid.org/0000-0001-7996-4109

\section{REFERENCES}

1 WHO: Geneva. Invest in eliminating hepatitis, 2020. Available: https:// www.who.int/news-room/detail/28-07-2019-invest-in-eliminatinghepatitis
2 Yang S, Wu J, Ding C, et al. Epidemiological features of and changes in incidence of infectious diseases in China in the first decade after the SARS outbreak: an observational trend study. Lancet Infect Dis 2017:17:716-25.

3 Wang P, Mao QA, Tao MX, et al. Survey on the status of health literacy of Chinese residents in 2008. Chinese $J$ Health Education 2010;26:243-6.

4 National Health Commission of the People's Republic of China. Publication on the National health literacy in Chinese population in 2017, 2020. Available: http://www.nhc.gov.cn/wjw/zccl/201809/e722 99ab37974d809b7e16b793763ded.shtml

5 China Internet Network Information Center. The 33rd China statistical report on Internet development, 2013. Available: http://www.cnnic. net.cn/hlwfzyj/hlwxzbg/hlwtjbg/201403/P020140305346585959798. pdf

6 China Internet Network Information Center. The 41st China statistica report on Internet development, 2020. Available: http://www.cnnic. net.cn/hlwfzyj/hlwxzbg/hlwtjbg/201803/P020180305409870339136. pdf

7 Zhang D, Han MJ, Chen QF, et al. The effect of using WeChat platform for college students to carry out health education of knowledge of AIDS. Chinese J AIDS \& STD 2016;22:550-2.

8 Chen CL, Shi CJ. Effect of WeChat health education model on nursing intervention of patients with thoracolumbar fracture treated with conservative therapy. Chinese and Foreign Medical Research 2019;17:173-6.

9 Qiu Y, Ren W, Liu Y, et al. Online health information in a rural residential population in Zhejiang Province, China: a cross-sectional study. BMJ Open 2019;9:e026202.

10 Nguyen HTL, Nakamura K, Seino K, et al. Association between a wider availability of health information and health care utilization in Vietnam: cross-sectional study. J Med Internet Res 2017;19:e405.

11 National Bureau of Statistics. National Bureau of statistics, 2017. Available: http://www.stats.gov.cn/tjsj/ndsj/2017/indexch.htm 